\title{
Application of Drones in the Whole Life Cycle of Engineering Construction Project
}

\author{
Houjie Qiu, ${ }^{1, *}$ \\ ${ }^{1}$ Shandong University of Science and Technology Resource College, Taishan, Tai'an, Shandong, China
}

\begin{abstract}
With the rapid development of information technology, unmanned aerial vehicle (UAV), as a new and popular technology, has also been tried in the field of engineering construction. In this paper, through the application of UAV technology in Australia's large-scale project to extinguish mountain fire, the application effect of UAV in the whole life cycle of engineering construction project is analogized. UAV assisted project progress, management quality and safety and other aspects, so that managers can timely and efficiently understand the situation of the project site, and effectively improve the management's control over the project construction, indicating the future application prospect of UAV technology in the construction field.
\end{abstract}

\section{Preface}

With the rapid development of information technology, a new round of industrial technology revolution has begun slowly. Emerging information technologies such as BIM, $3 \mathrm{D}$ printing and "Internet + " have emerged one after another. The traditional labor-intensive building mode has become technology intensive and knowledge intensive. As the pillar industry of our country, the construction industry has made considerable economic contribution. However, due to the complexity of the construction industry, although the speed of technical construction has been accelerated, the difficulty of managing technical construction has been further increased. The traditional construction management technology model can not meet the needs of effective management, especially in the planning, coordination, communication and other aspects of serious problems, leading to low efficiency of construction engineering operation. So, how to use the new information technology reasonably and effectively in the process of engineering construction management and make science and technology contribute to the effective project management has become a new problem.

\section{Introduction of UAV Technology}

UAV technology combines the flexibility and high speed of micro UAV platform, forming a unique UAV photography system, which can obtain complete and reasonable object information with high efficiency and low cost, and then process it through some computer software, and finally obtain the surveying and mapping products, which are combined with the actual site conditions, New methods and applications of project management are explored from different locations and texture details of buildings.
As a new data collection tool, UAV has the advantages of air operation and automation. In the application of engineering construction, it has the characteristics of "fast and efficient, small volume, simple operation, wide coverage and complete functions", which can realize realtime on-site monitoring. While improving efficiency, it can not only save labor and cost, it can also help manual workers in high-risk jobs.

With the wide production and promotion of civil 3D modeling UAV, the cost has also been reduced. All of these promote the civil 3D modeling technology to play a more important role in China's aviation, electric power, water conservancy, construction engineering, transportation and other important engineering construction. Application research has also begun to spread and expand to the fields of BIM, intelligent identification and other related technologies, and entered a new stage of technology integration and development. It can be used in many aspects, such as civil 3D modeling, deformation process monitoring, construction technology management, fire rescue and rescue, which greatly reduces the operation risk factor in construction, it can significantly improve the work efficiency. When the power transportation stringing, highway engineering across rivers, mountain areas and other special terrain, we can consider the use of automated unmanned aerial vehicles instead. [1]

\section{Application of UAV in fire fighting project in Australia}

By studying the application of UAV in Australian fire fighting engineering, it can be analogically applied to the whole life cycle of engineering construction projects.

In 2019, a mountain fire broke out in Australia's southeastern coastal and northwestern regions, causing extensive property damage, killing at least 30 people and about 1 billion wildlife. With the development of science

\footnotetext{
* Corresponding author: ${ }^{\mathrm{a} Z y x y t w @ s d u s t . e d u . c n}$
} 
and technology, in order to meet the needs of economy, safety and fire detection to the maximum extent, firefighters use unmanned aerial vehicle (UAV) for fire monitoring and situation awareness (SSA). SSA UAVs have high-definition thermal imaging cameras and telemetry sensors to monitor and report data from frontline personnel portable devices. SSA UAVs can help people monitor changing conditions so that the emergency operations center (EOC) can best guide operators to achieve the best results and maximum safety. UAV has simple structure and composition, it can be widely used in aerospace, three-dimensional photography, environmental detection and other fields, and has broad industrial prospects. Next, through the establishment of a reasonable model to study the application of UAV in the Australian fire.

\subsection{Simulation of fire distribution in ArcGIS}

First of all, we collected the Australian wildfire data from October 1, 2019 to January 7, 2020, then preprocessed the collected data, imported the processed data into ArcGIS, simulated the fire distribution in ArcGIS, described the local topographic map and fire characteristics through ArcGIS software, and drew the fire distribution map, then put the hovering radio relay unmanned aerial vehicle (UAV) position and divide the whole Victoria into equal areas, then use ant colony algorithm to establish a model to get the optimal route of SSA UAV patrol in each area, then analyze the sensitivity of the model, and when the shortest path is determined, then calculate the number and combination of UAVs and radio relay UAVs by linear programming, and finally output the optimal number and combination scheme of SSA UAVs and radio relay UAVs, and calculate the total cost.

We import the processed data into ArcGIS, simulate the fire distribution in ArcGIS, describe the local topographic map and fire characteristics through ArcGIS software, and draw the fire distribution map.

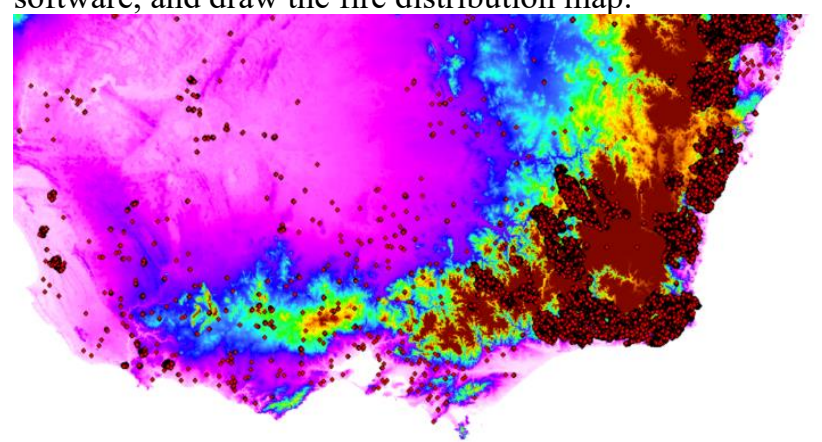

Figure 1: Wildfire distribution in Victoria, Australia

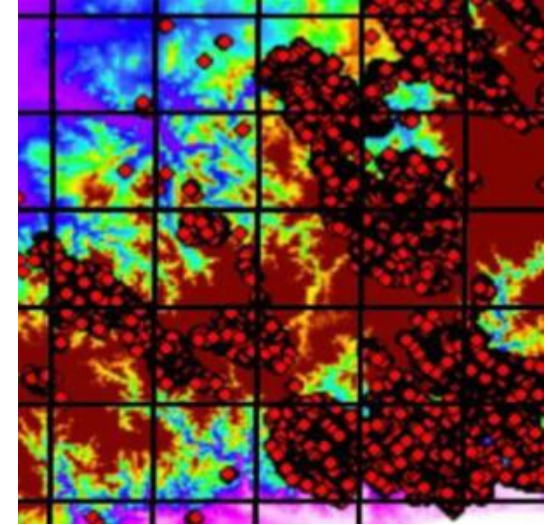

Figure 2: Example of divided areas

The red dots in the figure indicate the geographical location of the fire, and the denser the red dots indicate the greater the fire severity. We intercepted the fire distribution in Victoria in the fire distribution map of Australia. We divide many areas with length and width of 40 kilometers, covering the fire area. A radio relay drone is placed at the midpoint of each area.

\subsection{Using ant colony algorithm to solve the optimal route of SSA UAV}

It is known that the link lines corresponding to all necessary points are $\operatorname{len}_{i}(i=1,2, \cdots, d)$, according to a certain proportion coefficient $k$ After segmentation, new necessary points can be obtained:

$$
P_{i i}=\operatorname{len}_{i} \times k
$$

All link lines are discretized and dispersed, and the division length is as follows $\zeta$, the division number of each link line and the necessary points on the current link line ${ }^{i}$ Go to the necessary points on the next link line ${ }^{j}$ The probability of is:

$$
\begin{aligned}
& \pi_{i}=\left\{\begin{array}{l}
\operatorname{Int}\left(\frac{L_{i}}{\xi}\right), \operatorname{Int}\left(\frac{L_{i}}{\xi}\right)=2 k \\
\operatorname{Int}\left(\frac{L_{i}}{\xi}\right)+1, \operatorname{Int}\left(\frac{L_{i}}{\xi}\right)=2 k+1
\end{array}\right. \\
& P_{i, j}(t)=\left\{\begin{array}{l}
\frac{\left[\tau_{i, j}(t)\right]^{\alpha}\left[\eta_{i, j}(t)\right]^{\beta}}{\sum_{S \in I}\left[\tau_{i, s}(t)\right]^{\alpha}\left[\eta_{i, s}(t)\right]^{\beta}}, j \in I \\
0, j \notin I
\end{array}\right.
\end{aligned}
$$

In which, $\tau_{i, j}(t)$ As a necessary point $i$ To the necessary point $j$ The pheromone concentration between; $\eta_{i, j}(t)$ It is an heuristic function, which means that ants move from waypoints $i$ Transfer to waypoint $j$ The level of expectation, $\alpha$ It indicates the role of pheromone concentration in ants' path selection, $\beta$ Is the importance factor of heuristic function. I Represents the set of necessary points for ants to visit. After selecting the node, the pheromone will be updated: 


$$
\tau_{i, j}=(1-\rho) \bullet \tau_{i, j}+\rho \tau_{0}
$$

Among $0 \leq \rho \leq 1, \tau_{0}$ Is the initial value of the pheromone. When a complete search is finished, the pheromone should also be updated. The formula is the same as above, and will be $\tau_{0}$ replace with $\frac{1}{L_{\min }}$, in which $L_{\min }$ Is the shortest path value. [2]

\subsection{Sensitivity analysis}

In order to verify the accuracy of the model, this paper studies a part of fire hazard areas in New South Wales, and its fire distribution map is shown in the following figure.

The comparison curve between the shortest distance and the average distance of each generation is shown in the following figure.
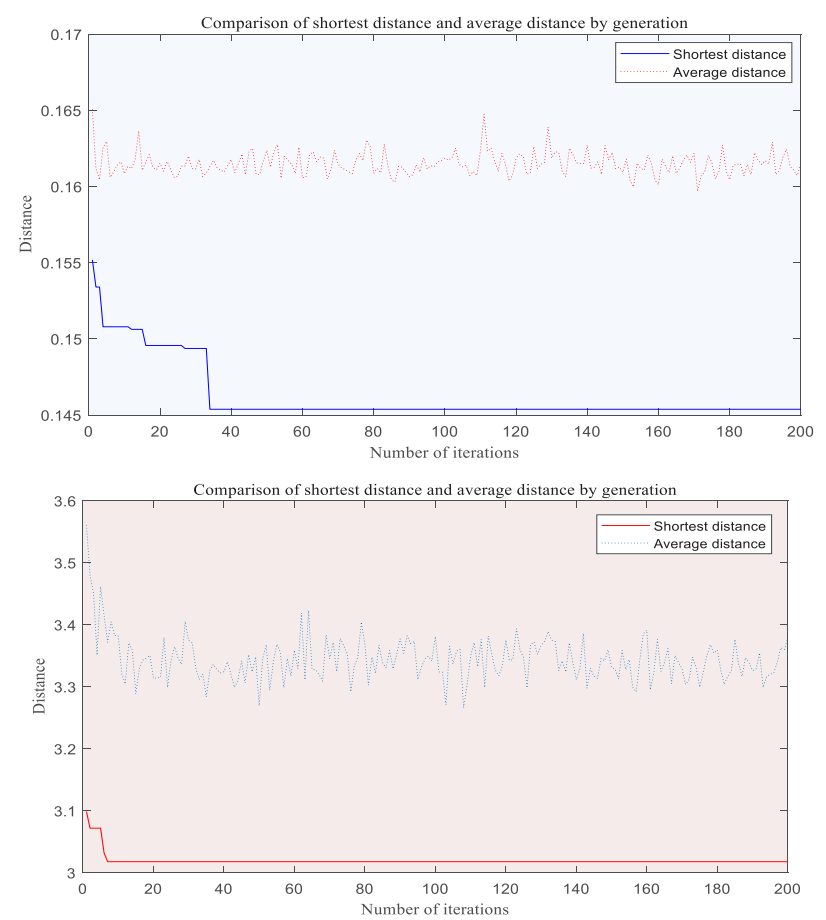

Figure 3: Comparison curve of shortest distance and average distance of each generation

Obviously, although the fire area and terrain conditions of the two regions are quite different, the trend of the comparison curves between the shortest distance and the average distance of each generation in the two regions is very similar, which shows that the model has good stability, low sensitivity and meets the requirements.

Firstly, the shortest path model of fire monitoring is constructed based on ant colony algorithm, and linear programming analysis is carried out. The final results show that:

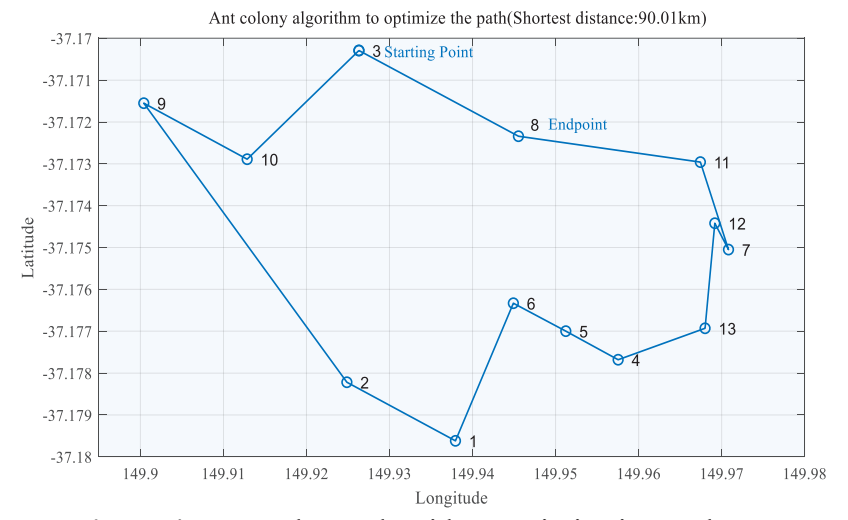

Figure 4: Ant Colony Algorithm Optimization Path

$\begin{array}{lllllll}\begin{array}{c}\text { The number of SSA in the unit } \\ \text { area }\end{array} & 1 & 2 & 3 & 4 & 5 \\ \begin{array}{c}\text { Number of partitions with } \\ \text { corresponding number of SSA }\end{array} & 13 & 33 & 42 & 27 & 11\end{array}$

Figure 5: Distribution of SSA UAVs

In a word, under the condition of combining UAV capability with safety and economy, considering the needs of observation and communication tasks and terrain, taking the size and frequency of fire events as parameters, the satellite is finally determined. The optimal number and combination of UAVs and radio relay UAVs are 368 SSA UAVs and 126 radio relay UAVs, with a total cost of AUD 4.94 million.

\section{Application and prospect of UAV in engineering construction field}

In the process of engineering construction, there are three stages of information collection: manual direct collection, semi-automatic collection by helicopter and unmanned aerial vehicle, and real-time automatic collection by sensors under the Internet of Things. The information collected by drones is characterized by strong timeliness and sharing, and the collection process is very convenient; the requirement on investment cost and use space is relatively low and very flexible. With the development of UAV technology, more and more UAVs are applied to the whole life cycle of construction projects, including early planning and decision-making, construction, use or operation, audit and other stages.

Early planning and decision-making stage: UAV has a wide field of vision, which is mainly used in project publicity, planning and design, engineering investigation and so on. In Australia's large-scale forest fire-fighting projects, firefighters use unmanned aerial vehicles to obtain complete and accurate information of ground objects at high efficiency and low cost in the early decision-making stage. In the engineering field, UAV can be used to design and widely publicize the early concept of real estate. Making various publicity materials from the perspective of unfinished buildings can directly and clearly show the potential visual effects of the scheme. Using UAV 3D imaging technology, high-precision realistic scene model of engineering survey can be 
established, which can be used for topographic mapping, geological identification and landslide estimation. In addition, based on the high-precision images of the surrounding environment provided by aerial photography projects, the UAV can also quickly acquire the original building information in the surrounding environment, including roads and water conservancy facilities. Thereby improving the efficiency of external condition investigation of engineering projects.

Construction stage: UAV can acquire image information during construction, collect point cloud data and create $3 \mathrm{D}$ models, which can be directly applied to auxiliary construction and site management. During the large-scale forest fire extinguishing project in Australia, firefighters carry unmanned aerial vehicles with various functions to carry out safety management, fire-fighting progress management and fire development trend supervision and management. In safety management and control, unmanned aerial vehicles can provide real-time images and location information of the location, and realize remote interaction between firefighters and command posts. In the field of engineering construction, the UAV three-dimensional real-time model is applied to large-scale deformation monitoring and engineering quantity calculation in the process of engineering construction, and the transformation of deformation monitoring and engineering quantity calculation from manual to automatic is completed. The remote interaction of personnel is to ensure the safety of personnel and machines. At the same time, the site shall be monitored in real time, so as to eliminate the danger over time and obtain the construction dynamic and recovery plan.

Use or operation stage: UAV can customize flight route, and combine with intelligent identification technology to help develop, maintain and repair, daily safety inspection and emergency management, including: automatic identification, positioning, monitoring and detection of pipelines, roads and other structures, so as to improve the monitoring efficiency of engineering structures after being put into use.

Audit stage: the construction project audit runs through the whole project construction period, and unmanned aerial vehicles are also playing a unique role, mainly including the verification of completion acceptance data in the auxiliary construction audit process, and the investigation of illegal and illegal buildings in the land audit. [1]

UAV aerial photography technology and 3D modeling technology can humanize the complete panorama, complete model and complete surveying and mapping data of construction projects. In carrying out the rectification and evidence collection of illegal buildings, using urban image data acquired by unmanned aerial vehicles, possible illegal buildings and their location information can be quickly identified, thus improving the efficiency of inspection and rectification of illegal buildings. [3]

In addition, the data and information obtained by UAV in the early stage of planning and decision-making, construction, use or operation are also very important, because these data and information can also be used as auxiliary materials for engineering audit, helping auditors to know the project situation in detail. Providing hidden information about the construction process and operation of the project will help to carry out the audit work efficiently in the future, the way of collecting audit information will also change.

\section{Conclusion}

Unmanned aerial vehicle (UAV) technology plays an important role in architectural and engineering applications, and has great application potential. UAV 3D imaging technology has the functions of fast translation, 3D model synthesis and continuous image data comparison. Based on the functions of UAV 3D imaging technology and the characteristics of UAV, the UAV 3D imaging technology has great application and development space in the field management during the construction stage. For example, it can visually present the real-time layout of the construction site, obtain the construction progress information of the actual project, and find the difference between the actual project and the design model by comparing the real life model with the BIM model. [4]

The environment of construction site is very complex and changeable, which is difficult to manage and costly in resources. Effective management and cost reduction are the core of construction companies. By studying the application of UAV technology in large-scale forest fire extinguishing projects in Australia, this paper compares the application effect of UAV in the whole life cycle of engineering construction projects. Combined with the actual situation of the construction site, the introduction of UAV technology into the construction site management will help speed up the construction progress and provide accurate information support for the construction site management. Although UAV technology still has defects in this respect, it's the advantage of effectively obtaining ground information may provide new ideas for construction site management.

\section{References}

1. Zhou, H., Hong, J.L., Lin, S.Z. (2019) Application of UAV technology in engineering construction. J. Journal of Engineering Management., 33: 9- 14.

2. Cheng, Q., Jing, T., Yu, Z.Y. (2016) UAV path planning based on ant colony optimization improved by cubic spline. J. Computer Measurement \& Control., 24: 272-274, 293.

3. Ma, W.A., Li, Y.Y., Sun, H., Chen, X.Q., Xie, C.R. (2021) Application of UAV technology in highway engineering construction management. J. Highway., 5: 78-79.

4. Ye, G., Xie, Y.X., Zhang, H.C., Cong, F.L. (2021) Application of drone technology in the field of construction engineering. J. Intelligent City., 2: 213217. 